\title{
RecQL1 DNA repair helicase: A potential tumor marker and therapeutic target against hepatocellular carcinoma
}

\author{
KAZUNOBU FUTAMI ${ }^{1}$, SACHIKO OGASAWARA ${ }^{2}$, HIDEYUKI GOTO ${ }^{1}$, \\ HIROHISA YANO ${ }^{2}$ and YASUHIRO FURUICHI ${ }^{1}$ \\ ${ }^{1}$ GeneCare Research Institute Co., Ltd., 19-2 Kajiwara, Kamakura, Kanagawa 247-0063; ${ }^{2}$ First Department of Pathology, \\ Kurume University School of Medicine, 67 Asahi-machi, Kurume, Fukuoka 830-0011, Japan
}

Received November 30, 2009; Accepted December 29, 2009

DOI: 10.3892/ijmm_00000375

\begin{abstract}
RecQL1 in the human RecQ DNA helicase family participates in DNA repair and recombination pathways in cell cycle replication. Immunohistochemical analysis of human hepatocellular carcinoma (HCC) tissues showed that RecQL1 expression is strongly correlated with histological grade and MIB-1 indices of HCC, and that the expression was greater in simple HCCs inducing extranodular growth or portal vein invasion than in HCCs not inducing extranodular growth or portal vein invasion. These histological data reveal the potential of RecQL1 as a biological marker predicting the malignancy and progression of liver cancer. High expression profiles were also produced by various HCC cells, including HCC cell lines established by us. When RecQL1 expression was silenced by siRNA in vitro, most HCC cells died of mitotic catastrophe. In a mouse orthotopic xenograft model of liver cancer with transplanted human HCC, RecQL1-siRNA mixed with cationic liposomes exhibited a strong anticancer effect that prevented the growth of the cancer. RecQL1-siRNA inhibited the growth of human HCC in the mouse liver, confirming that RecQL1 is an excellent molecular agent against liver cancer and suggests that RecQL1-siRNA formulated with liver-prone liposomes has excellent potential as a therapeutic drug against liver cancers.
\end{abstract}

\section{Introduction}

Human hepatocelullar carcinoma (HCC) is one of the most common tumors worldwide. The prognosis of HCC patients remains poor, and thus identifying useful therapeutic targets

Correspondence to: Dr Yasuhiro Furuichi, GeneCare Research Institute Co., Ltd., 19-2 Kajiwara, Kamakura, Kanagawa 247-0063 Japan

E-mail: furuichi@genecare.co.jp

Abbreviations: HCC, hepatocellular carcinoma; siRNA, small interfering RNA

Key words: hepatocelullar carcinoma, DNA damage, RNA interference, mitotic catastrophe, anti-cancer siRNA drug and prognostic markers are urgently required, although a dominant pathway has not been found responsible for the development of HCC (1). Liver-specific neoplasms arise under various physiological backgrounds and from virus infection that results in many types of cellular chromosome aberrations, gene mutations, epigenetic alterations and altered signaling pathways. Studies have shown that alterations of cell cycle regulators occur in HCC (2), particularly in structural or functional modifications in genes such as TP53, Rb1, P16/ INK4a and P21/WAF1, participating in the DNA damage response system (3-10).

The RecQL1 (also known as RecQL or RecQ1) helicase, a member of the RecQ DNA helicase family participating in genomic stability, is expressed highly in proliferating cells. Functionally, down-regulation of RecQL1 in HeLa cells by RNA interference increases sister chromatid exchanges (11). RecQL1-deficient mice produced by gene targeting are indistinguishable from wild-type mice except that embryonic fibroblasts from RecQL1-deficient mice are sensitive to ionizing radiation (12). These findings collectively indicate that the RecQL1 helicase suppresses chromosomal instability by participating in DNA repair during the cell cycle, but its function seems to be nonessential, since no human disease is known to be related to its gene mutation. Previously, we found that cancer cells were extremely sensitive to RecQL1 silencing by RNA interference with small interfering RNA (siRNA) and that cell growth was severely inhibited, as if these cancer cells were addicted to highly expressed RecQL1. Yet, noncancerous cells and all normal cells tested were unaffected by RecQL1-specific RNA interference $(13,14)$. This unique event was idenitfied as mitotic catastrophe during mitosis of the cell cycle in checkpoint system-deficient cancer cells due to accumulation of DNA damage caused by the absence of the RecQL1 helicase (14). To use this cancer cell-specific killing mechanism for the clinical benefit of anticancer chemotherapy, preliminary trials using model mice inoculated with various human cancer cells have been carried out (15).

In the present study, we focused our study on RecQL1 in hepatic cancers. First, we investigated the expression level of RecQL1 protein in clinically obtained HCCs ( 40 cases) to ascertain whether the expression level of the RecQL1 helicase is directly related to differentiation of HCC cells, malignancy of cancer cells and patient prognosis. Second, by using several HCC cell lines which included three HCC cell lines 
established in our laboratory (16-20), we studied the effect of RecQL1 silencing on cell proliferation in vitro. Third, we set up a mouse orthotopic hepatoma model with human HCC cells, and the anticancer efficacy of the RecQL1-siRNA/ cationic liposome complex was examined in vivo to assess whether RNA interference therapy is able to be used in the management of hepatic cancers. The results are discussed considering the possible use of RecQL1 expression as a developmental marker of hepatic cancer and of RecQL1siRNA as an anti-hepatic cancer drug.

\section{Materials and methods}

Pathological analysis of the expression of the RecQL1 helicase in human HCCs. Immunohistochemical examination of the RecQL1 helicase was performed by using paraffin sections of HCC and non-HCC tissues obtained with written consent from $40 \mathrm{HCC}$ patients who were surgically resected between 1994 and 1996 at Kurume University Hospital, Japan. The anti-RecQL1 antibody Q1N3, which was generated by us and was confirmed to be specific for the $\mathrm{N}$-terminal domain of RecQL1 helicase (amino acid residues 1-338) and the SignalAmplification System II (code K1497, Dako, Ely, UK) were used. The patients had no chemotherapeutic treatment before surgery. Four tissues were from hepatic B virus carriers, 27 tissues were from hepatic $\mathrm{C}$ virus carriers and 1 tissue was from a patient infected with both hepatic B and $\mathrm{C}$ viruses. HCC tissue samples consisted of 4 well to moderately differentiated HCCs, 28 moderately differentiated HCCs, 7 moderately to poorly differentiated HCCs, and 1 poorly differentiated HCC. The proliferation activity of HCC cells was monitored using Ki-67 expression as an MIB-1 index. For the evaluation standard of RecQL1, we used RecQL1positive bile duct epithelium in the same specimen (positive internal control). The specimen was evaluated as low expression (+) when it was stained as intensively as the positive internal control, as high expression $(++)$ when the staining was more intensive, and as negative (-) when it was not stained at all. We examined the relationship between RecQL1 expression and the clinicopathological features of the HCC cases, such as histological grade, MIB-1 index, tumor size, portal vein invasion and intrahepatic metastasis. Pathological features were evaluated according to the classification of the Liver Cancer Study Group of Japan. Statistical analyses were carried out for each clinicopathological characteristic among the $(-),(+)$ and $(++)$ groups.

The relationships between RecQL1 expression and histological grades, and between RecQL1 expression and the MIB-1 index were examined by using the JonckheereTerpstra test. The relationships between RecQL1 expression and recurrence or survival rate were evaluated by using the Fisher's exact P-test or by using the log-rank test, respectively. The relationships between RecQL1 expression and other clinicopathological features were examined by using the Kruskal-Wallis test.

Cells and cell culture conditions. HCC cell lines (HepG2, Hep3B, Huh7, SK-HEP-1, Li-7, HAK-1A, HAK-1B, KIM-1, KYN-1, KYN-2 and KYN-3) and normal cell lines ARPE19 (a normal spontaneously arising retinal pigmented epithelium cell line) and TIG-3 (normal lung diploid fibroblasts from human fetuses) were used. HepG2, Hep3B, SK-HEP-1, ARPE19 were obtained from ATCC (Manassas, VA, USA). Huh7 and Li-7 were obtained from Riken Cell Bank (Tsukuba, Japan). TIG-3 was obtained from the Japan Health Sciences Foundation (Osaka, Japan). The remaining six HCC cell lines were originally established by us from surgically resected HCC nodules at Kurume University, Japan. KIM-1 (16) and KYN-1 (17) were established from moderately differentiated HCC nodules, KYN-2 was established from moderately to poorly differentiated HCC nodules (18) and KYN-3 was established from peritoneal effusion of HCC patients with moderately to poorly differentiated HCC (19). HAK-1A and HAK-1B were established from a single HCC nodule showing a three-layered structure with a different histological grade in each layer (20). These cell lines were previously confirmed to retain the morphological and functional characteristics of the original tumor.

The cells were grown in Dulbeco's modified Eagle's or RPMI-1640 medium (Nacalai Tesque, Kyoto, Japan) and were incubated at $37^{\circ} \mathrm{C}$ in a humidified chamber supplemented with $5 \% \mathrm{CO}_{2}$. Some cells were treated with genotoxic agent doxorubicin (Adriamycin; Sigma, St. Louis, MO, USA) at the concentration $0.25 \mu \mathrm{M}$ for $24 \mathrm{~h}$ at $37^{\circ} \mathrm{C}$.

siRNA and RNA interference. siRNAs (21 bp) targeting RecQL1 mRNA (RecQL1-siRNA) and negative control siRNA (NS-siRNA) were chemically synthesized (Nippon EGT, Toyama, Japan). All siRNA sequences had an overhanging 3 '-dTdT at the 3 ' terminus (14). Sequence-specific gene silencing was confirmed by using cDNA microarray analysis with the Affymetrix GeneChip system (Human Genome U133 Plus 2.0 Array). For transfection at $24 \mathrm{~h}$ after plating, the cells were incubated with $40 \mathrm{nM}$ siRNA duplex for $8 \mathrm{~h}$ in the presence of RNAiMax (Invitrogen, Carlsbad, CA, USA) and were cultured in fresh medium.

Reverse transcriptase-PCR. After transfection, total RNA was extracted from cultured cells by using an RNeasy Mini Kit (Qiagen GmbH, Hilden, Germany) according to the manufacturer's protocol. Reverse transcriptase-PCR analyses were carried out by using the ABI PRISM 7000 Sequence Detection System with TaqMan probes and primers (ABI, Foster, CA, USA). The $\beta$-actin gene was used as the internal standard (TaqMan probe ID; 431088E; ABI). RecQL1 silencing was monitored by using a specific primer set (Hs00262956_m1). Expression of genes P16/INK4a (CDKN2A), Hs00233365_m1; P21/WAF1 (CDKN1A), Hs00355782_m1; P27/KIP1 (CDKN1B), Hs00153277_m1; TP53; Hs00153340_m1; and Rb1, Hs01078066_m (ABI) were also determined and were used as references.

Cell proliferation assays. Cell proliferation was measured by using colorimetric assays based on the cleavage of tetrazolium salt WST-8 (Nacalai Tesque). The absorbance of the formazan dye formed was measured at $450 \mathrm{~nm}$ at $3 \mathrm{~h}$ after adding the reagent.

Flow cytometric analysis. Trypsin-treated cells were washed with phosphate-buffered saline (PBS) and were fixed in ice- 
cold methanol for $2 \mathrm{~h}$. The cells were treated with pancreatic RNase A (Nippon Gene, Toyama, Japan) and stained with propidium iodide (Sigma) for $30 \mathrm{~min}$. The cells were then analyzed by using flow cytometry. Fluorescence was measured by using Epics XL (Beckman Coulter K.K., Tokyo, Japan). For each sample, 10,000 events were analyzed.

Immunoblotting. The protein levels of RecQL1 and other proteins participating in the cellular DNA damage repair system were monitored by using immunoblot analysis. The cells were washed with ice-cold PBS, were pelleted and then were lysed in a sodium dodecyl sulfate (SDS) buffer containing $1 \%$ SDS, $2 \%$ B-mercaptoethanol, 20\% glycerol, $30 \mathrm{mM}$ Tris- $\mathrm{HCl}$ (pH 6.8) and 0.2 M dithiothreitol. The cell lysate was boiled for $10 \mathrm{~min}$ and then was electrophoresed on 5-20\% gradient SDS-polyacrylamide gels. Proteins fractionated on the gels were electrophoretically transferred to polyvinylidene difluoride membranes (Immobilon, Millipore, MA, USA) and were blocked overnight with $5 \%$ skimmed milk in PBS. The membranes were then incubated with either anti-RecQL1 monoclonal antibody Q1N3 or anti- $\beta$-actin monoclonal antibody (ICN Biomedicals, Aurora, OH, USA) for $60 \mathrm{~min}$ at room temperature. Antibodies detecting TP53 (DO-1), P21/WAF1 (Ab-1), PCNA and Rb1 proteins were purchased from Santa Cruz Biotechnology (Santa Cruz, CA, USA), Oncogene (San Diego, CA, USA), Abcam Limited (Cambridge, UK) and Cell Signaling Technology (Danvers, MA, USA), respectively, and were used to detect the proteins important in oncology. The membranes were washed with $0.05 \%$ Tween-20 in PBS, incubated with anti-mouse IgG conjugated with horseradish peroxidase (DakoCytomation, Carpinteria, CA, USA) and washed. They were subsequently developed using an enhanced chemiluminescence reagent (ECL Plus, Amersham Biosciences, UK).

Orthotopic implantation in mice. Mice (BALB/c nut/+) purchased from Charles River Japan, Inc. (Yokohama, Japan) were maintained in a pathogen-free environment. They received humane care in accordance with the National Institutes of Health Guide for the Care and Use of Laboratory Animals.

The orthotopic hepatoma model of mice was designed according to the method of Lu et al (21). Briefly, KYN-2 cells $\left(2.0 \times 10^{6}\right)$ were harvested from subconfluent cultures and were suspended in serum-free medium containing $50 \%$ Matrigel (BD Bioscience, Bedford, MA, USA). The cells were slowly injected into the mouse liver through the diaphragmatic surface by using a 28 -gauge needle. The skin was then closed with an absorbable suture and sterile surgical clips. On day 7 after the cell injections, mice with established intrahepatic tumors were randomized into three groups (each group $n=10-11$ ) and received therapeutic treatments of the siRNA/cationic liposome complex from day 7 .

Intravenous injection of the RecQL1-siRNA/LIC-101 complex. The RecQL1-siRNA/LIC-101 complex was prepared by Nippon Shinyaku Co., Ltd. (Kyoto, Japan) as previously described (22). The RecQL1-siRNA/LIC-101 complex (2 mg siRNA/kg mouse body weight, equivalent to $\sim 50 \mu \mathrm{g}$ siRNA/mouse) in $100 \mu \mathrm{l}$ of $10 \%$ (w/v) maltose solution, was injected intravenously once on days 7, 9, 11, 14, 16, 18, 21, 23, $25,28,30$ and 32 , a total of 12 injections. The anticancer effects were evaluated on day 35 . The average liver weights were analyzed by using the Dunnett's test.

\section{Results}

RecQL1 expression in various HCCs and the relationship to clinicopathological MIB-1 features of HCC. RecQL1 was expressed to various degrees in most HCC nodules ( $82.5 \%$ of the $40 \mathrm{HCC}$ specimens) (Fig. 1A and B). RecQL1 expression was high around the peripheral area of the HCC nodules in some cases. Tumor thrombi in the portal vein showed relatively strong RecQL1 expression. In non-cancerous areas, the RecQL1 expression was also observed in infiltrating lymphocytes, endothelial cells and hepatocytes to various degrees (Fig. 1C and D). The degree of RecQL1 expression was evaluated as negative (-) in 7 (3 well to moderately differentiated, 4 moderately differentiated; $17.5 \%$ ), low expression $(+)$ in 15 ( 1 well to moderately differentiated, 13 moderately differentiated, 1 moderately to poorly differentiated; $37.5 \%$ ), and high expression (++) in 18 (11 moderately differentiated, 6 moderately to poorly differentiated, 1 poorly differentiated; 45.0\%) HCC cases (Table I). Upon analysis of the relationship between RecQL1 expression and clinicopathological features (Table I, Fig. 2), we found that RecQL1 expression was: i) significantly correlated with histological grade and MIB-1 indices of the HCCs; ii) significantly higher in the simple nodular type HCC cases (with extranodular growth) than in the HCCs without extranodular growth; iii) significantly higher in the HCC cases with portal vein invasion than in the HCCs without portal vein invasion; and iv) higher in the HCC nodules of diameter $\geq 2.0 \mathrm{~cm}$ than in the HCC nodules of diameter $<2.0 \mathrm{~cm}$. RecQL1 expression and other clinicopathological features, such as prognosis, including recurrence and survival time of patients, showed no apparent relationship.

RecQL1 helicase expression levels in various HCC cell lines. RecQL1 expression in various HCC cell lines was investigated. As controls, normal cells ARPE19 and TIG-3 were similarly characterized. Most HCC cells expressed a greater amount of RecQL1 helicase protein than the normal cells (Fig. 3A). RecQL1 expression was exceptionally high in the Li-7, SK-HEP-1, HAK-1A, HAK-1B, KYN-2 and KYN-3 HCC cell lines.

To understand the expression of other genes participating in DNA repair and the cell cycle, HCC cells were treated with genotoxic agent doxorubicin and the induced proteins were characterized (Fig. 3A). TP53, which is a DNA damage sensor, was not expressed in Hep3B, Li-7, KIM-1 and KYN-2 cells in the absence of doxorubicin, nor after doxorubicin treatment [TP53 vs. TP53 (Dox)] (Fig. 3A). In the KYN-1 cells, TP53 expression remained high, even in the absence of doxorubicin, suggesting that the TP53 gene mutates to an inactive form and so may not be able to contribute to genomic stability. The TP53 gene mutates in HCC cells KYN-1, KYN-3, HAK-1A and HAK-1B (20) and it mutates in SK-HEP-1 and Huh7 cells, but induction of the TP53 expression after DNA damage seems to be unaffected (23). In the present study, 

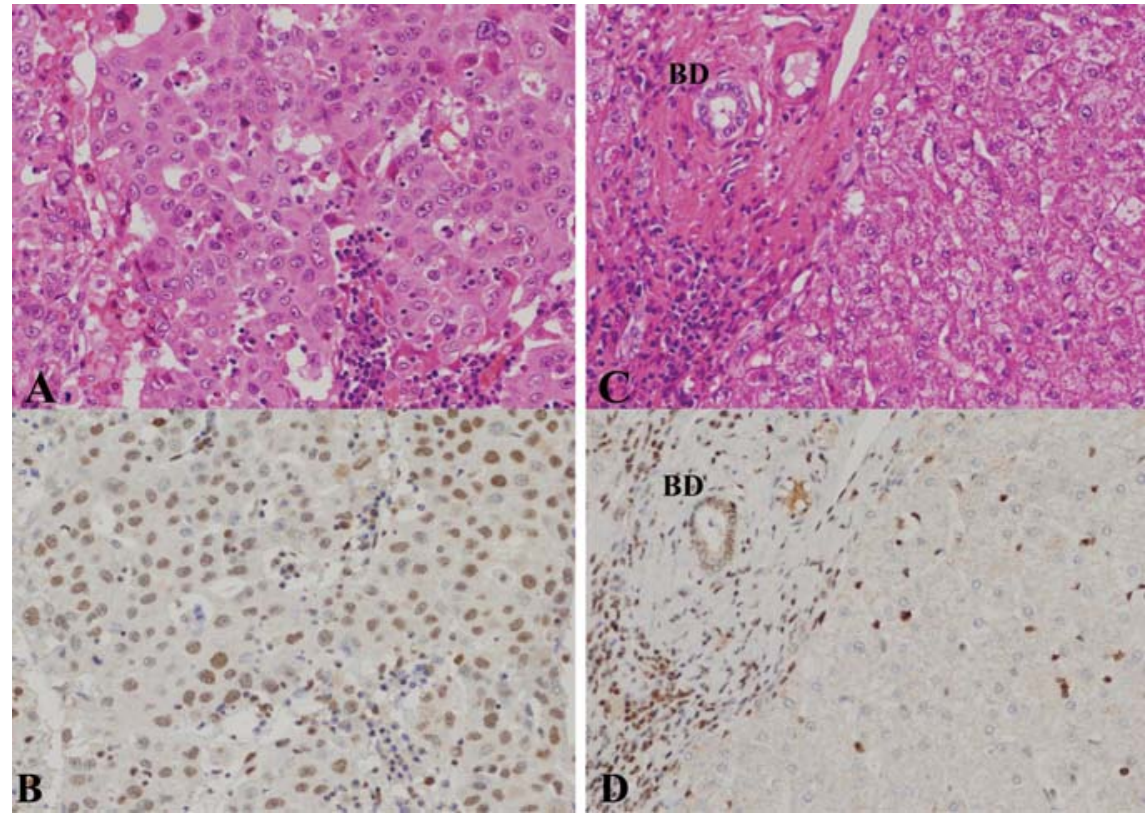

Figure 1. Immunohistochemical staining of RecQL1 in HCC and non-HCC tissues. (A) Moderately to poorly differentiated HCC stained with hematoxylin and eosin (H\&E) (x100). (B) RecQL-1 expression in the nuclei of HCC cells (x200). (C) non-HCC tissue with a bile duct (BD) in the portal tract (H\&E) (x100). (D) RecQL-1 expression in bile duct epithelial cells and infiltrating lymphocytes in the portal tract, and endothelial cells in the sinusoid (x100).

TP53 gene expression appeared normal in HepG2 cells, as the TP53 expression was induced highly by doxo-rubicin treatment. In the normal cells, APRE19 and TIG-3, TP53 expression increased in response to DNA damage caused by the doxorubicin treatment.

Next, we investigated P21/WAF1 gene expression and its response to DNA damage by doxorubicin. The P21/WAF1 gene was expressed poorly in most HCC cells, except HepG2 and SK-HEP-1 cells, but it was highly expressed in ARPE19 and TIG-3 normal cells, indicating that P21/WAF1 may be a candidate HCC-specific tumor marker. P21/WAF1 is induced in cells containing wild-type TP53 when the cells are exposed to DNA damaging agents, but not in cells containing the mutant TP53. These data indicate that most of the HCC cell lines that we investigated contained an incomplete DNA repair system that otherwise should be activated by the TP53P21/WAF1-mediated DNA damage signaling pathway. Also, $\mathrm{Rb} 1$ was not expressed in Hep3B cells. It should be emphasized that TP53, P21/WAF1 and Rb1 were expressed efficaciously in normal cells in response to DNA damage by doxorubicin (Fig. 3A), while they were not in most, if not all, HCC cell lines.

mRNA expression levels of TP53, Rb1, P16/INK4a and P21/WAF1 were compared among the various HCC cell lines by using reverse transcriptase-PCR analysis. TP53 was poorly expressed in Hep3B, Li-7, KIM-1 and KYN-2 cells, $\mathrm{Rb} 1$ was not expressed well in Hep3B cells, and the P16/ INK4a gene was not expressed in Li-7 and SK-HEP-1 cells. Again, the P21/WAF1 mRNA expression was low in most, if not all, HCC cell lines and was consistent with the results from the Western blot analysis (Fig. 3A and B). The data clearly indicate that the G1/S checkpoint system was defective in these proliferating $\mathrm{HCC}$ cells, consistent with previous studies (2-10).

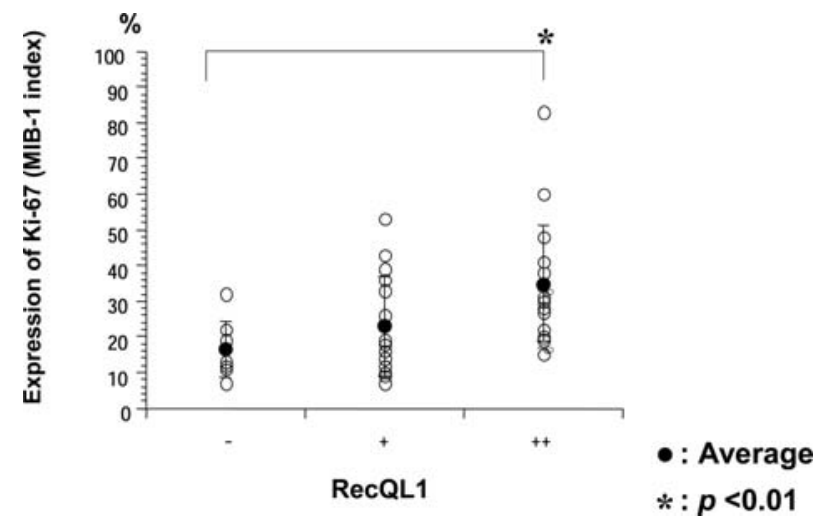

Figure 2. Relationship between RecQL1 and Ki-67 expression (MIB-1 index) in HCC. MIB-1 indices were compared in three different groups: RecQL1 negative group, RecQL1 low expression group, and RecQL1 high expression group. Their respective average MIB-1 indices were 16.6, 23.1 and $34.9 \%$. Their averages are indicated by closed circles. A significant difference in MIB-1 indices was observed between the RecQL1 negative and RecQL1 high expression groups.

Induction of mitotic catastrophe in HCC cell lines by RecQL1 silencing. We investigated the effect of RecQL1 silencing in these HCC cell lines that lacked an intact DNA damage sensor and checkpoint system but replicated actively. To our surprise, RecQL1-siRNA killed a wide range of proliferating $\mathrm{HCC}$ cell lines efficaciously after it induced mitotic catastrophe. Fig. 4A shows the typical features of mitotic catastrophe observed in the KYN-3 cells. The KYN-3 cells clearly began to die as RecQL1 was silenced by siRNA treatment (Fig. 4C), resulting in accumulation of cells arrested at the $\mathrm{M}$ phase.

To define this cell-killing effect by RecQL1 silencing, time course experiments were performed with several HCC 
Table I. RecQL1 expression and clinicopathological features in HCC.

\begin{tabular}{|c|c|c|c|}
\hline RecQL1 expression & - & + & ++ \\
\hline \multicolumn{4}{|l|}{ Histological grade ${ }^{a}$} \\
\hline Well + Mod & 3 & 1 & 0 \\
\hline Mod & 4 & 13 & 11 \\
\hline Mod + Poor & 0 & 1 & 6 \\
\hline Poor & 0 & 0 & 1 \\
\hline \multicolumn{4}{|l|}{ MIB-1 index $(\%)^{\mathrm{a}}$} \\
\hline$<14$ & 4 & 5 & 0 \\
\hline $14-27$ & 2 & 5 & 6 \\
\hline $28-51$ & 1 & 4 & 10 \\
\hline$>51$ & 0 & 1 & 2 \\
\hline \multicolumn{4}{|l|}{ Tumor size } \\
\hline$\leq 2 \mathrm{~cm}$ & 2 & 2 & 1 \\
\hline$>2 \mathrm{~cm}$ & 5 & 13 & 17 \\
\hline \multicolumn{4}{|l|}{ Macroscopic type } \\
\hline Simple nodular type & 7 & 12 & 8 \\
\hline $\begin{array}{l}\text { Simple nodular type }{ }^{\mathrm{b}} \\
\text { with extranodular growth }\end{array}$ & 0 & 3 & 10 \\
\hline \multicolumn{4}{|l|}{ Capsule formation } \\
\hline$(-)$ & 1 & 1 & 1 \\
\hline$(+)$ & 6 & 14 & 17 \\
\hline \multicolumn{4}{|l|}{ Capsular invasion } \\
\hline$(-)$ & 2 & 1 & 1 \\
\hline$(+)$ & 5 & 14 & 17 \\
\hline \multicolumn{4}{|l|}{ Portal vein invasion } \\
\hline$(-)$ & 5 & 10 & 6 \\
\hline$(+)^{\mathrm{c}}$ & 2 & 5 & 12 \\
\hline \multicolumn{4}{|l|}{ Intrahepatic metastasis } \\
\hline$(-)$ & 5 & 13 & 11 \\
\hline$(+)$ & 2 & 2 & 7 \\
\hline \multicolumn{4}{|l|}{ Non-cancerous tissue } \\
\hline Chronic hepatitis & 3 & 9 & 13 \\
\hline Liver cirrhosis & 4 & 6 & 5 \\
\hline
\end{tabular}

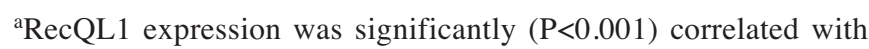
histological grade and MIB-1 indices of HCC cells. ${ }^{b} \mathrm{P}<0.01$ vs. the simple nodular type. ${ }^{c} \mathrm{P}<0.05$ vs. (-). Well, well-differentiated; Mod, moderately differentiated; Poor, poorly differentiated.

cell lines of various genetic backgrounds. The cells were incubated once with $40 \mathrm{nM}$ siRNA and were cultured in siRNA-free medium for several days. HCC cell lines, such as SK-HEP-1, KYN-2 and KYN-3, began to die after a lag-time of about $48 \mathrm{~h}$ (Fig. 4B). In contrast, the growth of normal ARPE19 and TIG-3 cells that rapidly proliferated similar to HCC cell lines was almost unaffected despite RecQL1 gene silencing by RecQL1-siRNA (Fig. 4B). A slight retardation

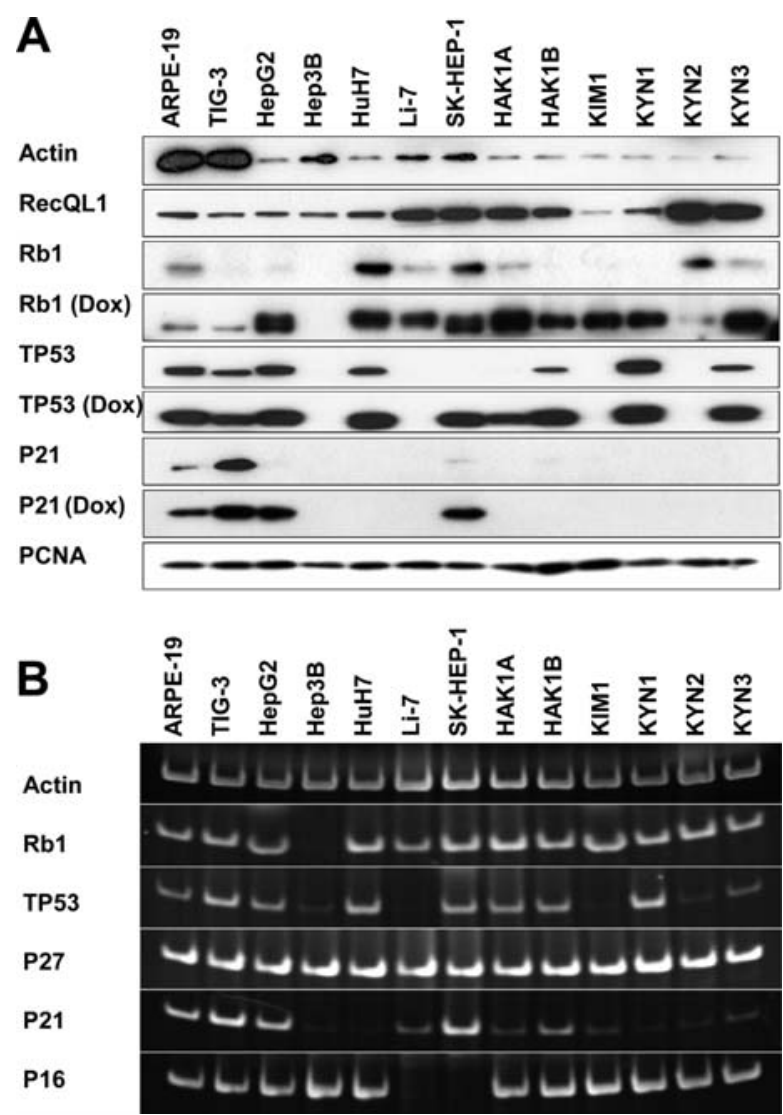

Figure 3. Expression of RecQL1 and cell cycle-related regulation genes in HCCs. (A) Cell lysates $(10 \mu \mathrm{g})$ were applied to each lane. Proteins underwent Western blot analysis by using individual antibodies. The cellular induction of gene expression upon DNA damage was monitored by protein analysis before and after doxorubicin (Dox) treatment. (B) Quantitative analysis of gene expression by the determination of individual mRNAs with reverse transcriptase-PCR.

(10-15\%) of growth was noted in these normal cell lines when compared with the non-silencing siRNA-treated cells.

Flow cytometric cell analysis showed a large peak correlated with the subG1 fraction representing debris associated with cell death in all RecQL1-siRNA-treated HCC cells. Some RecQL1-siRNA-treated HCC cells underwent unequal cell division after the arrested $\mathrm{M}$ phase, resulting in giant cells that could not divide and that looked like senesced cells (data not shown). This suggested that RecQL1-siRNAtreated HCC cells entered the M phase with no regulation and then developed mitotic catastrophe or aberrant cell division. In contrast, the normal cells, ARPE19 and TIG-3, showed no mitotic catastrophe because of premature arrest and repair at both the G1 and G2 phases. Non-silencing siRNA treatment under the same conditions did not affect the cell cycle progression of HCC and normal cells, indicating that cancer cell-specific mitotic catastrophe is dependent on RecQL1 silencing. Also, cancer cell-specific mitotic catastrophe is clearly dependent on replication of cells and genetic defects in HCC cells, such as the absence of P21/ WAF1 expression.

Effect of systemic administration of the RecQL1-siRNA/ LIC-101 complex in the KYN-2 orthotopic xenograft model in mice. We investigated the therapeutic efficacy of RecQL1- 
A
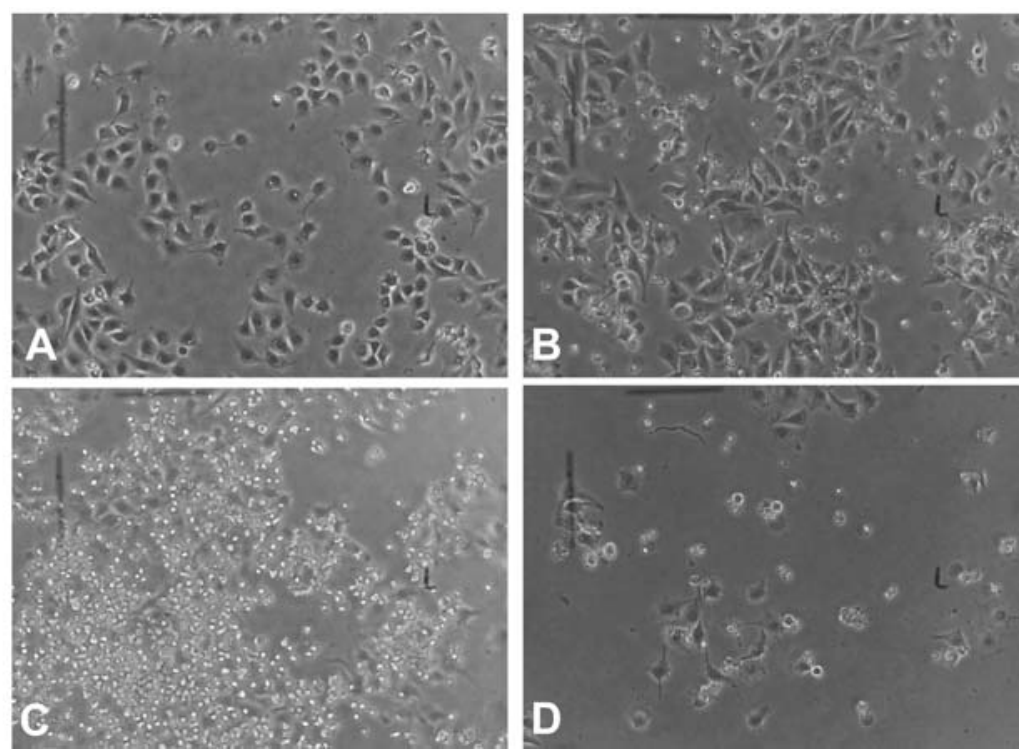

B
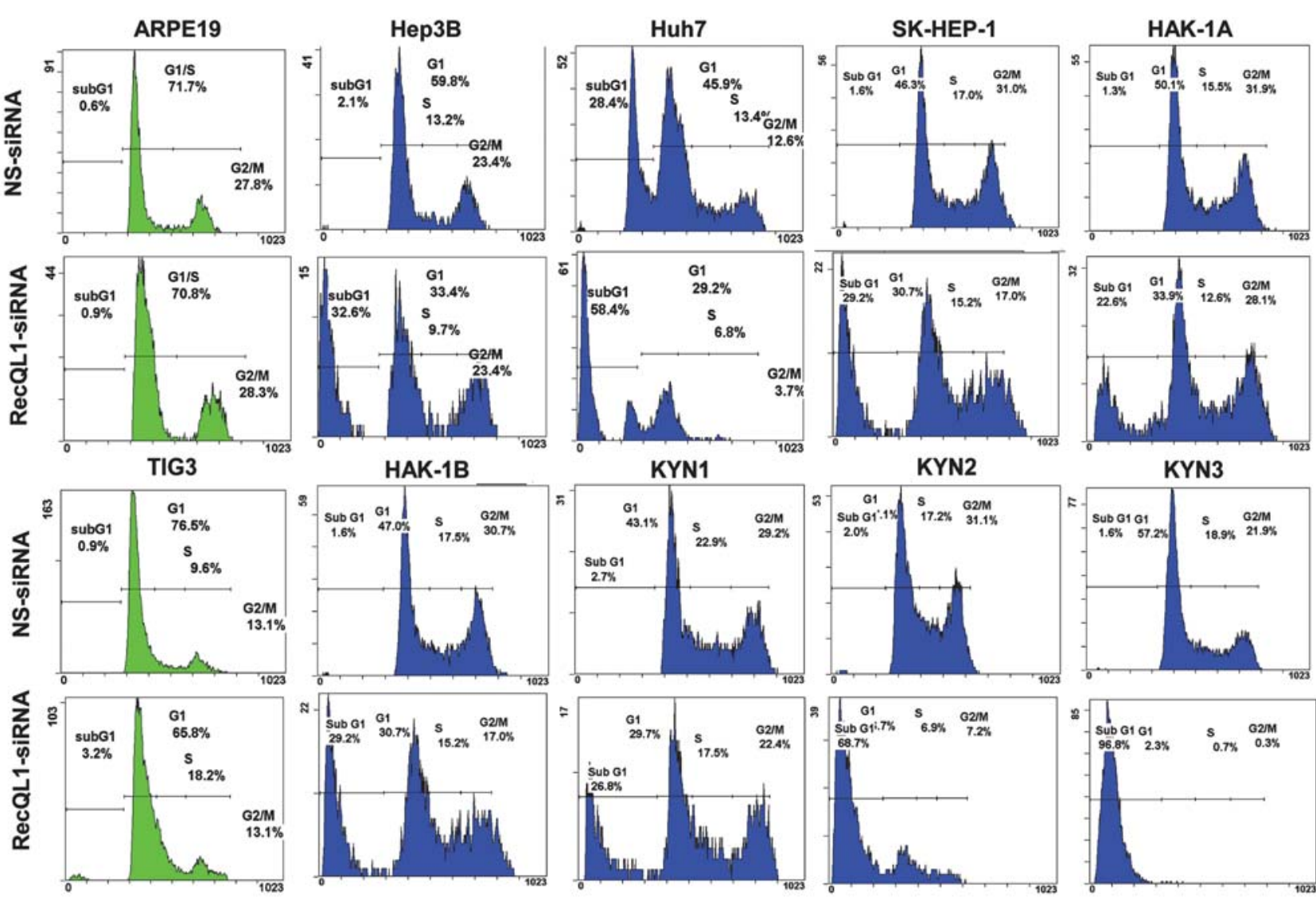

Figure $4 \mathrm{~A}$ and $\mathrm{B}$.

siRNA against KYN-2 cells in an orthotopic hepatoma model by using cationic liposome LIC-101 that carries siRNA to the cytosol of liver cells (22). In the in vivo experiments, the RecQL1-siRNA/LIC-101 complex was administered to mice which had formed KYN-2 nodules. Fig. 5A shows the KYN-2 tumors formed in the livers, and the results of therapeutic treatment with systemic injection of the RecQL1-siRNA/ LIC-101 complex. KYN-2 tumors grew to a large volume in control mice that were injected with $10 \%$ maltose solution (Fig. 5B). KYN-2 tumors grew extensively in the liver of mice injected with the non-silencing siRNA/LIC-101 complex (Fig. 5C). However, only a few KYN-2 tumors appeared in the liver of mice treated with the RecQL1-siRNA/LIC-101 complex (Fig. 5D), indicating that RecQL1-siRNA effectively prevented the growth of KYN-2 tumors in the orthotopic hepatoma model. A clear statistical difference $(\mathrm{p}<0.05)$ in liver weight was observed between mice injected with $10 \%$ maltose or the non-silencing siRNA/LIC-101 complex and mice injected with the RecQL1-siRNA/LIC-101 complex (Fig. 5B). 


\section{Normal cell lines}
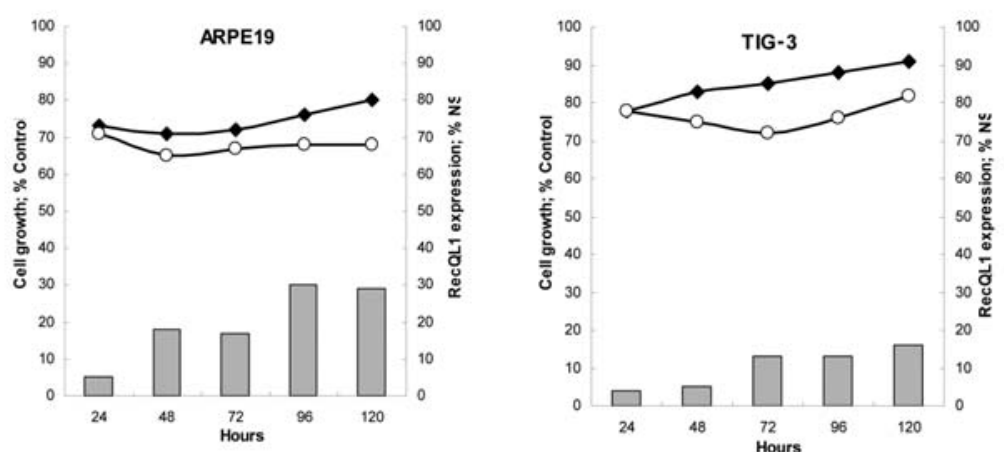

\section{Cancer cell lines}
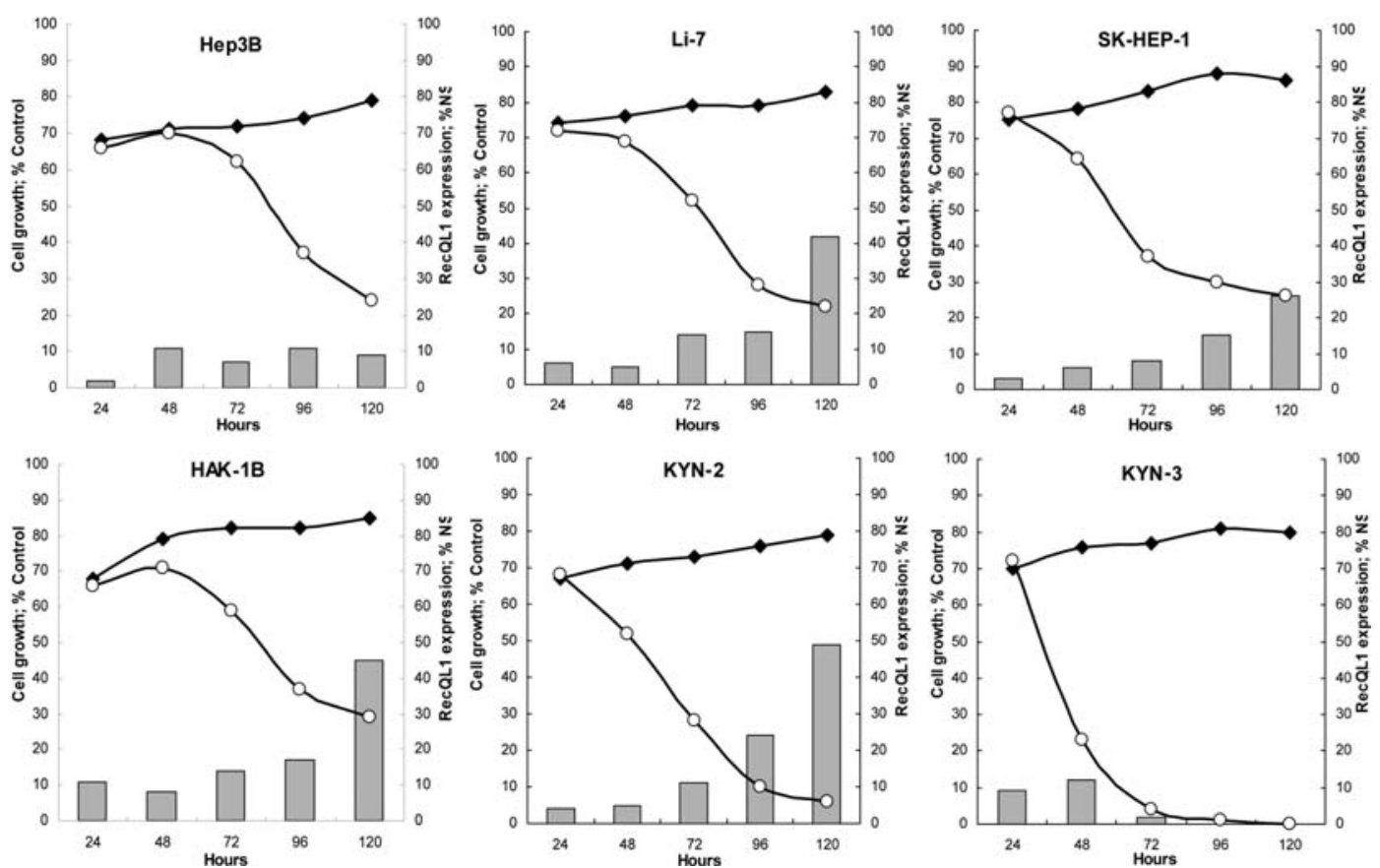

Figure 4. Growth inhibitory effect of RecQL1-siRNA on various HCC cells. (A) KYN-3 cells treated with RecQL1-siRNA. Microscopic features of cells were monitored at A, 24 h; B, 36 h; C, 48 h; and D, 72 h. (B) Flow cytometric analysis of HCC cells 72 h after siRNA treatment. (C) Time course analysis of viability of HCC cells and RecQL1 silencing. Six HCC cell lines (Hep3B, SK-HEP-1, LI-7, HAK-1B, KYN-2 and KYN-3) and two normal cell lines (TIG-3 and ARPE19) were transfected either with RecQL1-siRNA (open circle) or with NS-siRNA (solid circle). The viability of cells is shown as the relative growth to that of non-treated cells. The histograms show the relative expression level of RecQL1-silenced cells compared with the NS-siRNA-treated cells used as controls.

\section{Discussion}

Increased expression of RecQL1 in undifferentiated human HCC cells. The RecQL1 helicase has long been known to participate in DNA repair reactions, but little is known about its relation to human disease and the clinical outcome of therapy. Li et al (24) previously showed that single nucleotide polymorphisms of RecQL1 have a marked influence on the clinical response to therapy using genotoxic drugs and radiation, and to the overall survival of patients with pancreatic cancer. In this study, we investigated RecQL1 expression in clinical specimens of HCC and found that RecQL1 expression was significantly correlated with histological grade and MIB-1 indices of HCC development. RecQL1 expression and the MIB-1 index had a close relationship, suggesting that RecQL1 participates in cell proliferation, and, importantly, the RecQL1 expression profile resembled that of the well- known tumor marker PCNA of previous studies $(25,26)$. These data verify that RecQL1 expression may be a reliable tumor marker, similar to PCNA, representing the progression and malignancy of cancers.

Incomplete regulation of the cell cycle in human HCC cells. HCC cells have a divergent and incomplete cell cycle regulation system that is perhaps compensated by a high expression of DNA repair enzymes to guarantee rapid and competent proliferation of cells (Fig. 3). In most of the HCC cell lines, expression of TP53 and P21/WAF1, which responds to DNA damage signaling and regulates cell cycle progression by inhibiting the cyclin-CDK complex, respectively, was severely reduced. This reduction also causes the inability of $\mathrm{Rb} 1 / \mathrm{E} 2 \mathrm{~F}$ to reduce the transcription of genes promoting the cell cycle, resulting in the inability of G1/S arrest during cell cycle progression (27). Conceivably, 

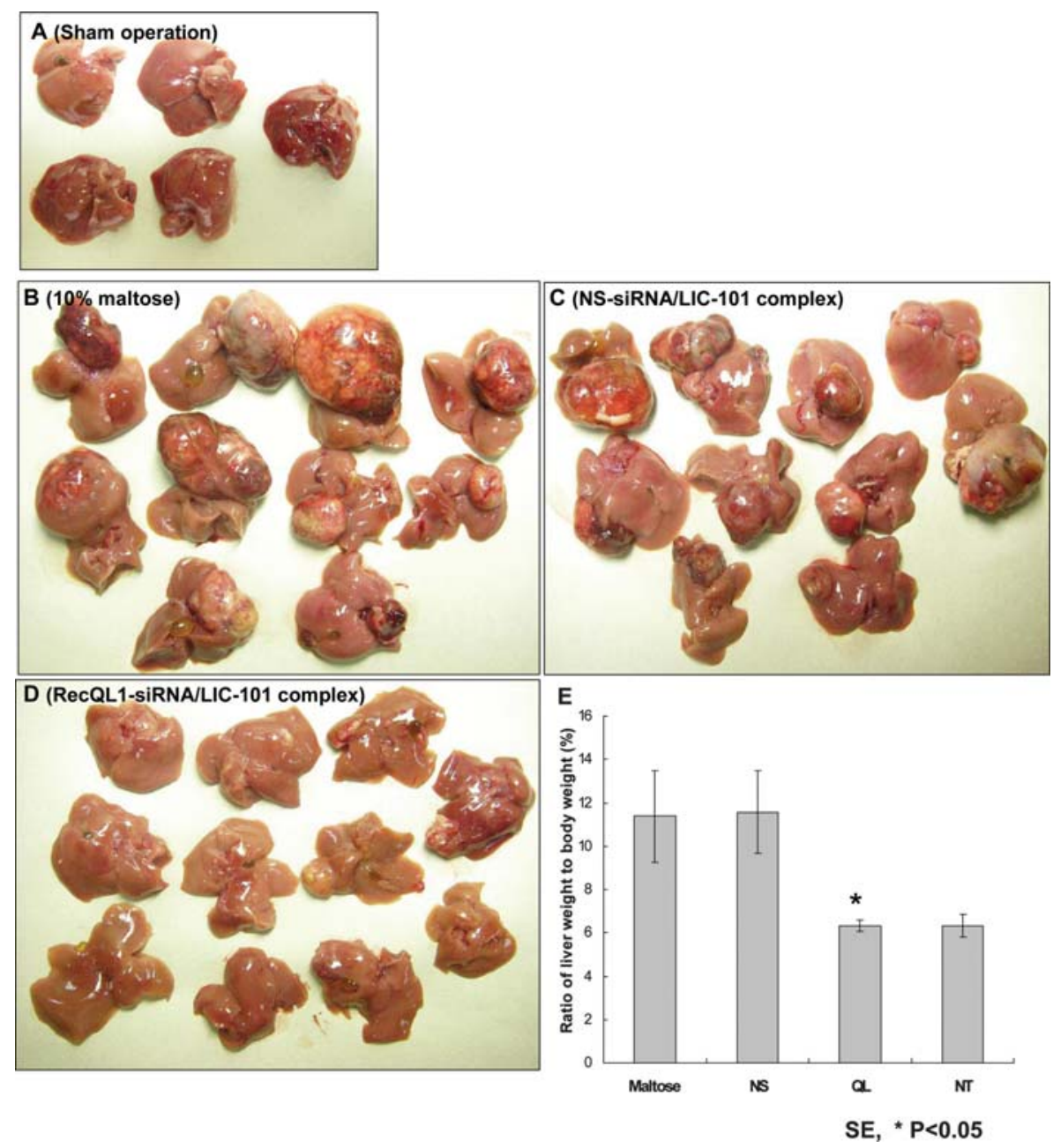

Figure 5. Therapeutic anticancer activity of RecQL1-siRNA in a mouse xenograft model of HCC. siRNA (2 mg/kg) formulated with LIC-101 cationic liposomes (suspended in $10 \%$ maltose) was injected intravenously into the tail vein of mice. (A) Sham operation mouse livers, (B) mouse livers treated with $10 \%$ maltose, (C) mouse livers treated with the NS-siRNA/LIC-101 complex, (D) mouse livers treated with the RecQL1-siRNA/LIC-101 complex, and (E) ratio of the weight of the liver to the weight of one mouse. Bars, the standard error of the measurements. *RecQL1-siRNA/LIC-101 complex is statistically more effective than the NS-siRNA/LIC-101 complex.

the absence of P21/WAF1 expression prevented the G1/S cell cycle stop due to lack of Rb1/E2F transcription initiation. These cell cycle defects found so far in many types of cancer cells exist in HCC, as shown in this study. Even in SK-HEP-1 cells in which the expression of P21/WAF1 appeared to be normal, P16/INK4a which is required to stop cell cycling, was not expressed (Fig. 3B). Here, the promoter inactivation by DNA methylation might have caused the absence or diminution of P16/INK4a expression as previously reported for various types of cancer cells $(5,6)$.

Cancer cell-specific mitotic catastrophe induced by RecQL1 silencing. RecQL1 helicase is highly up-regulated in rapidly proliferating cancer cells and in other transformed cells (28), suggesting that the RecQL1 helicase is needed for genomic stability in rapidly proliferating cells. In this study, we showed that RecQL1-siRNA killed various HCC cell lines by inducing mitotic catastrophe. However, the normal cell lines, TIG-3 fibroblastic cells and APRE19 epithelial cells (Fig. 4), as well as the WI38 and HUVEC cells were all tolerant to RecQL1 silencing. By contrast, checkpoint-defective cancer cells were sensitive because they failed to stop the cell cycle in order to correct the DNA damage caused by the absence of RecQL1. Notably, P21/WAF1, which is generally induced by TP53 and is important for cell cycle arrest, decreased in many of the HCC cells. Retrovirus integration into host chromosomes and computational simulations have shown that a single double-strand DNA break formed during replication is sufficient to kill cells deficient in DNA repair (29). In cancer cells, mitotic catastrophe due to DNA damage is most probably avoided by highly up-regulated repair enzymes such as RecQL1 that coordinate well with DNA replication to repair DNA damage. HCC cell death caused by silencing the RecQL1 helicase is thus consistent with the findings of Daniel et al (29).

Anticancer activity of RecQL1-siRNA in vivo in an orthotopic model of HCC xenograft in mice. The in vitro experiment showed that specific cancer cell death can be achieved efficiently by RecQL1 silencing (Fig. 5). RecQL1-siRNA mixed with liver-prone LIC-101 liposomes showed excellent anti-hepatic cancer activity in a mouse orthotopic hepatoma 
model (Fig. 5). This anticancer activity was dependent on several cell conditions related to each other as special features of cancer cells: i) high expression of the RecQL1 helicase, ii) active replication of cells, and iii) an impaired checkpoint function that permits cells with DNA damage to enter the M phase. Normal hepatic cells do not have these features and thus they do not show mitotic catastrophe caused by RecQL1 silencing. We propose the systemic administration of the RecQL1-siRNA/LIC-101 complex as an effective therapeutic system against hepatic cancer due to the expected absence of patient drug-associated adverse effects.

\section{Acknowledgements}

We thank Ms. Chie Ito for the technical assistance and Professor Masamichi Kojiro of Kurume University School of Medicine for the discussion and encouragement. We also thank Professor Shigeki Arii and Dr Arihiro Aihara of the Tokyo Medical and Dental University for the helpful discussions.

\section{References}

1. Llovet JM and Bruix J: Molecular targeted therapies in hepatocellular carcionoma. Hepatology 48: 1312-1327, 2008.

2. Ozturk M: Genetic aspects of hepatocellular carcinogenesis. Semin Liver Dis 19: 235-242, 1999.

3. Elmore LW, Hancock AR, Chang SF, et al: Hepatitis B virus X protein and p53 tumor suppressor interactions in the modulation of apoptosis. Proc Natl Acad Sci USA 94: 14707-14712, 1997.

4. Greenblatt MS, Feitelson MA, Zhu M, et al: Integrity of p53 in hepatitis B X antigen-positive and -negative hepatocellular carcinomas. Cancer Res 57: 426-432, 1997.

5. Chaubert P, Gayer R, Zimmermann A, et al: Germ-line mutations of the p16INK4 (MTS1) gene occur in a subset of patients with hepatocellular carcinoma. Hepatology 25: 1376-1381, 1997.

6. Hui AM, Sakamoto M, Kanai Y, et al: Inactivation of p16INK4 in hepatocellular carcinoma. Hepatology 24: 575-579, 1996.

7. Furutani M, Arii S, Tanaka S, et al: Decreased expression and rare somatic mutation of the CIP1/WAF1 gene in human hepatocellular carcinoma. Cancer Lett 111: 191-197, 1997.

8. Hui AM, Kanai Y, Sakamoto M, Tsuda $H$ and Hirohashi S: Reduced p21WAF1/CIP1 expression and p53 mutation in hepatocellular carcinomas. Hepatology 25: 575-579, 1997.

9. Kuroki T, Fujiwara Y, Nakamori S, Imaoka S, Kanematsu T and Nakamura Y: Evidence for the presence of two tumorsuppressor genes for hepatocellular carcinoma on chromosome 13q. Br J Cancer 72: 383-385, 1995.

10. Zhang X, Xu HJ, Murakami Y, et al: Deletions of chromosome $13 \mathrm{q}$, mutations in retinoblastoma 1 , and retinoblastoma protein state in human hepatocellular carcinoma. Cancer Res 54: 4177-4182, 1994.

11. LeRoy G, Carroll R, Kyin S, Seki M and Cole MD: Identification of RecQL1 as a Holliday junction processing enzyme in human cell lines. Nucleic Acids Res 33: 6251-6257, 2005.
12. Sharma S, Stumpo DJ, Balajee AS, et al: RecQL, a member of the RecQ family of DNA helicases, suppresses chromosomal instability. Mol Cell Biol 27: 1784-1794, 2007.

13. Sharma S and Brosh RM Jr: Human RecQ1 is a DNA damage responsive protein required for genotoxic stress resistance and suppression of sister chromatid exchanges. PLoS One 12: e1297, 2007.

14. Futami K, Kumagai E, Makino H, et al: Induction of mitotic cell death in cancer cells by small interference RNA suppressing the expression of RecQL1 helicase. Cancer Sci 99: 71-80, 2008.

15. Futami K, Kumagai E, Makino H, et al: Anticancer activity of RecQL1 helicase siRNA in mouse xenograft models. Cancer Sci 99: 1227-1236, 2008

16. Murakami T: Establishment and characterization of human hepatoma cell line (KIM-1). Acta Hepatol Jpn 25: 532-539, 1984.

17. Yano H, Kojiro $M$ and Nakashima T: A new human hepatocellular carcinoma cell line $(\mathrm{KYN}-1)$ with a transformation to adenocarcinoma. In Vitro Cell Dev Biol 22: 637-646, 1986.

18. Yano H, Maruiwa M, Murakami T, et al: A new human pleomorphic hepatocellular carcinoma cell line, KYN-2. Acta Pathol Jpn 38: 953-966, 1988.

19. Murakami T, Maruiwa M, Fukuda K, Kojiro M, Tanaka M and Tanikawa K: Characterization of a new human hepatoma cell line (KYN-3) derived from the ascites of the hepatoma patient (Abs.). Proc 47th Annual Meeting Jpn J Cancer Res p292, 1988.

20. Yano H, Iemura A, Fukada K, Mizoguchi A, Haramaki M and Kojiro M: Establishment of two distinct human hepatocellular carcinoma cell lines from a single nodule showing clonal differentiation of cancer cells. Hepatology 18: 320-327, 1993.

21. Lu YS, Kashida Y, Kulp SK, et al: Efficacy of a novel histone deacetylase inhibitor in murine models of hepatocellular carcinoma. Hepatology 46: 1119-1130, 2007.

22. Yano J, Hirabayashi K, Nakagawa S, et al: Antitumor activity of small interfering RNA/cationic liposome complex in mouse models of cancer. Clin Cancer Res 10: 7721-7726, 2004.

23. Bressac B, Galvin KM, Liang TJ, Isselbacher KJ, Wands JR and Ozturk M: Abnormal structure and expression of p53 gene in human hepatocellular carcinoma. Proc Natl Acad Sci USA 87: 1973-1977, 1990 .

24. Li D, Frazier M, Evans DB, et al: Single nucleotide polymorphisms of RecQ1, RAD54L, and ATM genes are associated with reduced survival of pancreatic cancer. J Clin Oncol 24: 1720-1728, 2006.

25. Kitamoto M, Nakanishi T, Kita S, et al: The assessment of proliferating cell nuclear antigen immunohistochemical staining in small hepatocellular carcinoma and its relationship to histologic characteristics and prognosis. Cancer 72: 1859-1865, 1993.

26. Adachi E, Hashimoto $\mathrm{H}$ and Tsuneyoshi M: Proliferating cell nuclear antigen in hepatocellular carcinoma and small cell liver dysplasia. Cancer 72: 2902-2909, 1993.

27. El-Deiry WS, Harper JW, O'Connor PM, et al: WAF1/CIP1 is induced in p53-mediated G1 arrest and apoptosis. Cancer Res 54: 1169-1174, 1994.

28. Kawabe T, Tsuyama N, Kitao S, et al: Differential regulation of human RecQ family helicases in cell transformation and cell cycle. Oncogene 19: 4764-4772, 2000.

29. Daniel R, Litwin S, Katz R and Skalka AM: Computational analysis of retrovirus-induced SCID cell death. J Virol 75: 3121-3128, 2001. 